folg einwenden können, dass in den meisten Kommunen eine Vergabe der Impftermine nach dem Wohnortprinzip beschränkt werde. $\mathrm{Ob}$ eine solche Vorgehensweise grundsätzlich zulässig ist, kann hier offenbleiben. Allerdings könnte eine entsprechende Regelung bei summarischer Prüfung aus den vorgenannten Gründen zumindest nicht rückwirkend und anspruchsbeseitigend in schon begonnene zweistufige Impfungen eingreifen. Die Knappheit von Impfstoffen war darüber hinaus keine neue, überraschende Tatsache, sondern der ASt. von Anfang an bekannt; zumal der Bundesgesetzgeber gerade vor dem Hintergrund der nur unzureichend verfügbaren Impfstoffe die CoronaImpfV erlassen hatte.

Es sind bei summarischer Prüfung auch keine sonstigen Änderungen der Sach- oder Rechtslage erkennbar, die die Selbstbindung der Ag. und somit den Anspruch der ASt. nachträglich hätten entfallen lassen können. Eine Änderung der Sach- oder Rechtslage ist - wie ausgeführt - bei summarischer Prüfung nicht ersichtlich. Auch hätte die Stornierung des zweiten Impftermins voraussichtlich keinen wirksamen Widerruf der Zusage ( $\$ 38$ Abs. 2 VwVfG analog i. V.m. $\int 49$ Abs. 2 Nr. 5 VwVfG) dargestellt. Denn ein schwerer Nachteil für das Gemeinwohl hätte bei summarischer Prüfung schon deshalb nicht vorgelegen, weil die Interessen der ASt. denen der Allgemeinheit und auch denen der Einwohner Magdeburgs gleichwertig gewesen wären. Es dürfte überdies im Interesse der Allgemeinheit sein, dass möglichst viele ,impfwillige“ Personen auch schnellstmöglich gegen das Corona-Virus geimpft werden, um eine effektive Eindämmung des Virus erreichen zu können.

Die ASt. hätten voraussichtlich auch das Vorliegen eines Anordungsgrundes glaubhaft gemacht.

Sie haben insbesondere mit der vorgelegten eidesstattlichen Versicherung v. 9.6.2021 glaubhaft gemacht, dass sie sich erfolglos anderweitig um Termine für die Zweitimpfung bemüht haben.

Ihnen hätten danach nach aller Voraussicht ohne eine Entscheidung nach $\$ 123$ Abs. 1 S. 2 VwGO zu ihren Gunsten besonders schwerwiegende Nachteile gedroht, nämlich die Beeinträchtigung bzw. der Wegfall des Impfschutzes und die weitere Folge einer nichtabsehbaren Möglichkeit des erneuten Beginns mit einer zweistufigen Impfung.

Die von den Antragstellern nicht unmittelbar nach der telefonischen Vereinbarung eines Impftermins in Haldensleben, sondern nach Durchführung der zweiten Impfung abgegebene Erledigungserklärung ist unter Berücksichtigung der Bedeutung der Zweitimpfung für die ASt. auch als umgehend zu bewerten.

$[\ldots]$

https://doi.org/10.1007/s00350-022-6129-6

\section{Anmerkung VG Magdeburg, Beschl. v. 13.7.2021 - 1 B 120/21 MD}

\section{Marius Hossbach und Niklas Hossbach}

\section{Bedeutung}

Vor dem Hintergrund der,,vierten Welle“ und voraussichtlich flächendeckenden Impfungen, werden die hier aufgeworfenen Rechtsfragen auch künftig bedeutsam sein.

Rechtsanwalt Dr. iur. Marius Hossbach,

Fachanwalt für Medizinrecht und Mitglied der Ethik-Kommission der Ärztekammer Hamburg, Korten Rechtsanwälte AG,

Neuer Wall 44, 20354 Hamburg, Deutschland

und von Dr. iur. Niklas Hossbach, Referendar am OLG Celle, Schloßplatz 2, 29221 Celle, Deutschland
Mehr als das hat die Angelegenheit eine besonders emotionale politische Facette: An der Sache entbrannte kurz vor der Landtagswahl in Sachsen-Anhalt (6. 6. 2021) abermals der alte Ost-West-Konflikt: Es schlugen sich mehrere Landes- und Lokalzeitungen in Niedersachsen und Sachsen-Anhalt auf jeweils eine der Seiten. Der Oberbürgermeister von Magdeburg schaltete sich verständnislos ein (,moralisch zutiefst verwerflich“ [die Zweitimpfung in Magdeburg einzufordern]). Den Betroffenen wurde ein "Impftourismus“ auf Kosten des Nachbarlandes vorgeworfen. Niedersachen hatte zu jener Zeit eine leicht höhere Impfquote hatte als SachsenAnhalt.

\section{Rechtliche Bewertung}

\section{Ist die Bestätigungs-E-Mail eines Impftermins bereits eine verbindliche Zusicherung?}

Das Gericht erkennt zutreffend, dass vorliegend die Bestätigungs-E-Mail des Impftermins noch keine bindende Selbstverpflichtung der Behörde als Zusicherung gem. $\$ 38$ Abs. 1 S. 1 VwVfG (Instruktiv zur Zusicherung Schröder, in: Schoch/Schröder, Juli 2020, \38 VwVfG, Rdnr. 13) zur Durchführung der Impfung darstellt und mithin selbst noch keinen Anspruch auf Durchführung der Impfungen begründet. Erforderlich wäre ein objektiv erkennbarer Rechtsbindungswille. Die Behörde muss unzweifelhaft erkennen lassen, dass sie sich zum angekündigten Handeln verpflichten möchte (Stelkens, in: Stelkens/Bonk/Sachs, 9. Aufl. 2018, § 38 VwVfG, Rdnr. 21). Dies ist vorliegend unter dem Vorbehalt des Disclaimers auf der Homepage des Impfzentrums, zunächst die persönlichen Voraussetzungen zur Impfung überprüfen zu wollen, nicht anders zu verstehen, $\iint_{133}$, 157 BGB. So sollte insb. die Identität und Priorisierung überprüft werden. Dies ist praxisgerecht, um die Einhaltung der gesetzlich vorgegebenen Impfreihenfolge einhalten zu können.

Trotzdem ist in der Bestätigungs-E-Mail - offenbar entgegen des VG - eine verbindliche Zusicherung zu sehen. Durch diese verpflichtete sich das Impfzentrum eindeutig erkennbar dahingehend, zunächst die persönlichen Voraussetzungen zu überprüfen und nur bei Nichtvorliegen der Voraussetzungen von der Impfung abzusehen. Dafür streitet ferner das Indiz, dass das zukünftige Handeln hinsichtlich Ort und Zeit des Impftermins bereits konkret umschrieben wurde (Schröder, in: Schoch/ Schröder, Juli 2020, \$38 VwVfG, Rdnr. 14). Im Umkehrschluss hat sich das Impfzentrum bei Vorliegen der aufschiebenden Bedingung (Prüfung der Identität und der Priorisierung der zu impfenden Person) zur Impfung verpflichtet. Insbesondere das Schriftformerfordernis des \$38 Abs. 1 S. $1 \mathrm{VwVfG}$ ist gewahrt. Insofern hat die Buchungsbestätigung sehr wohl eine rechtliche Qualität, unabhängig von der vorbehaltenen Überprüfung der persönlichen Voraussetzungen. Im konkreten Fall hat das VG zwar richtig erkannt, dass eine Zusicherung vorlag, es zumindest für die Erstimpfung offengelassen, worin diese letztlich zu sehen ist.

An eine solche Zusicherung ist die Behörde nur dann nicht mehr gebunden, sofern sich nach Abgabe der Zusicherung die Sach- und Rechtslage derart ändert, dass die Behörde bei Kenntnis der nachträglich eingetretenen Änderungen eine Zusicherung nicht gegeben hätte oder aus rechtlichen Gründen nicht hätte geben dürfen, $₫ 38 \mathrm{Abs} .3$ VwVfG. Die Zusicherung erfolgte in zulässiger Weise. Auch haben sich vorliegend die tatsächlichen Umstände nicht derart geändert. Ungeachtet der rechtlichen Qualität der vorliegenden „Stornierung“, hat das Gericht zu Recht erkannt, dass die Anforderungen an einen Widerruf der Zusicherung hier nicht gegeben waren. 
Im vorliegenden Fall hätte eine „Stornierung“ gegen Art. 3 Abs. 1 GG verstoßen. Der Wunsch der Antragsgegnerin, den ca. 1.000 Niedersachsen die sonst übliche Zweitimpfung zu verwehren und die Impfung nunmehr eigenen Einwohnern zukommen zu lassen, wäre eine ungerechtfertigte Ungleichbehandlung. Selbst wenn allein auf die Interessenlage Magdeburgs abgestellt worden wäre, so stünde ein Verfall der Erstimpfung für Bürger aus der Nachbarregion einer Erstimpfung in Magdeburg gegenüber. Eine weitere ungerechtfertigte Ungleichbehandlung lag in dem Umstand, dass die Zweitimpfung den ca. 1.500 Nichtmagdeburgern aus Sachsen-Anhalt nicht ,storniert“ wurde.

\section{Ist vom Impfanspruch nur die Erst-} oder auch die Zweitimpfung umfasst?

Das Gericht stellt für den Zeitpunkt der Zusicherung für die Zweitimpfung offensichtlich auf die Herausgabe der Zweittermins direkt im Anschluss an die Erstimpfung ab. Dies impliziert, dass es grundsätzlich für die Erstund Zweitimpfung jeweils gesonderte Zusicherungen bedarf und in der Konsequenz sich das Impfzentrum jeweils auch nur zur Erst- bzw. Zweitimpfung verpflichten würde.

Obgleich es im Fall nicht darauf ankam, ob bereits die erste Zusicherung beide Impfungen umfasst oder ob es einer zweiten Zusicherung für die zweite Impfung bedarf, verkennt das VG offenbar, dass es einer zweiten Zusicherung für die Zweitimpfung nicht bedurfte. Abermals ist anhand der Definition der Zusicherung entscheidend, wie der objektive Erklärungswert der Bestätigungs-E-Mail zu verstehen ist, $\iint 133,157 \mathrm{BGB}$.

Denn mit der Nennung des Termins zur Zweitimpfung wurde diese zwar vorbehaltlos, ,zugesichert“. Doch umfasst bereits die ursprüngliche Bestätigungs-E-Mail die Zusicherung zur Erst- und Zweitimpfung als „Paket“. Dass bei der Bestätigung des Erstimpftermins nicht bereits der Zweitimpftermin genannt wird, liegt nicht am fehlenden Willen zur Zweitimpfung, sondern daran, dass die Lieferungen der exakten Impfmengen nicht tagesgenau Wochen zuvor vorhergesagt werden konnten. Es ist allgemein bekannt, dass erst mit Erhalt der Erstimpfung, unaufgefordert der Termin für die Zweitimpfung vergeben wird, wie es auch hier der Fall war. Dies ergibt sich bereits aus dem Umstand, dass Impfzentren oder Hausärzte zu diesem Zeitpunkt keine isolierten Erst- oder Zweitimpfungen anboten.

Subsidiär besteht ein Teilhabe- und Leistungsanspruch auf die Zweitimpfung aus Art. 2 Abs. 2 S. 1 GG i. V. mit Art. 1 Abs. 1 S. 2 GG. Zum Schutz der individuellen und kollektiven Gesundheit sind die vom RKI vorgegebenen Impfabstände von den staatlichen Institutionen zwingend einzuhalten. Andernfalls droht ein zeitnaher Verlust des aufgebauten Impfschutzes und mithin die Verfehlung des Impfziels. Alles andere wäre nicht geeignet, diesem staatlichen Auftrag zu entsprechen. Im Ergebnis hat das VG dies zutreffend erkannt.

\section{Fazit}

Der im Ergebnis begrüßenswerte Beschluss schafft nur teilweise Rechtssicherheit in einem hochsensiblen Thema, das Millionen Bürger betrifft. Zugleich zeigt sich, dass sich Impfzentren in einem anspruchsvollen Rechtsumfeld bewegen. Unbürokratisches und schnelles Agieren in Krisenzeiten ist zwar allgemein erwünscht, wie z.B. dass kurzfristig überschüssige Vakzine rasch verwendet werden, erhöht jedoch auch die Gefahr der fehlerhaften Rechtsanwendung. Ob des Konfliktfeldes bleibt daher zu hoffen, dass die juristische Gemengelage nicht zu einem zurückhaltenden und womöglich langsameren Impftempo führt.

\section{Aufwändige Diagnostik und Notfallbehandlung bedingen keine stationäre Behandlung bei Weiterverlegung in ein anderes Krankenhaus}

\author{
SGB V §§2 Abs. 1, 12 Abs. 1, 39
}

1. Eine aufwändige Diagnostik und Notfallbehandlung im Schockraum eines Krankenhauses begründet nicht bereits die spezifische Eingliederung in das Versorgungssystem eines Krankenhauses.

2. Diese Form der Behandlung ist bei Weiterverlegung in ein anderes Krankenhaus als ambulante Notfallbehandlung zu qualifizieren, welche aus der Gesamtvergütung $z u$ finanzieren ist.

3. Eine stationäre Aufnahme im notfallmäßig behandelnden Krankenhaus findet dabei regelhaft nicht statt, ebenso wenig wie eine Aufnahmeentscheidung.

4. Auch ein anderweitiger Vergütungsanspruch des Krankenhauses scheidet aus, wenn die Aufnahmeuntersuchung nicht von der ambulanten Notfallbehandlung zu unterscheiden ist.

5. Eine Einweisung durch den Rettungsdienst ist mit einer vertragsärztlichen Verordnung von Krankenhausbehandlung nicht vergleichbar. (Leitsätze des Bearbeiters)

BSG, Urt. v. 18. 5.2021 - B 1 KR 11/20 R (LSG für das Saarland)

Problemstellung: Obgleich das LSG für das Saarland in der nun vom 1. Senat des BSG ,kassierten“ Entscheidung nachvollziehbar und rechtlich einwandfrei herausgestellt hatte, warum im vorliegenden Fall ein auskömmlicher Vergütungsanspruch des Leistungserbringers für eine (kurze) vollstationäre Behandlung vorlag, wird der 1. Senat des BSG in seiner doch recht neuen Besetzung ohne Not und in nicht nachvollziehbarer Weise die Diskussion um die Vergütung der Notfallbehandlung wieder bzw. weiter anfachen.

Irritierenderweise führt das BSG zunächst selbst aus, dass die ambulante Notfallbehandlung nicht ,trennscharf" von der Aufnahmeuntersuchung abzugrenzen sei, meint dann aber, dass trotz der erfolgten Intubation und Beatmung im Schockraum des erstbehandelnden Krankenhauses keine Aufnahmeuntersuchung, sondern eine ambulante Notfallbehandlung erfolgt sei. Dies ist umso erstaunlicher, als dass das LSG für das Saarland in der angegriffenen Entscheidung nachvollziehbar dargelegt hatte, dass in tatsächlicher Hinsicht das Krankenhaus nicht als Notfallambulanz gehandelt habe, sondern vielmehr eine intensivmedizinische Intervention durchgeführt wurde, die nur mit den Mitteln eines Krankenhauses und in einer besonderen Einrichtung der Notfallmedizin (nicht der Notfallambulanz) vorgenommen werden konnten.

Es ist daher auch unverständlich, dass sich das BSG in einem obiter dictum dazu auslässt, dass selbst eine kurze Aufnahme auf der Intensivstation, welche ggf. vor der Verlegung in das andere Krankenhaus notwendig geworden wäre, einen Anspruch auf vollstationäre Vergütung nicht hätte entstehen lassen, da eine solche Aufnahme, gegen das Wirtschaftlichkeitsgebot [...] verstoßen" hätte. Diese Auffassung dürfte das BSG exklusiv für sich beanspruchen, da der Schockraum - selbst wenn man ihn (wie vermutlich allein das BSG) nicht als Teil der Krankenhausversorgung qualifiziert - nur der Erstversorgung dient. Insofern ist es fernliegend, etwa bei einer wegen eines anderweitigen Bedarfs des Schockraums notwendigen Aufnahme auf der Intensiv-

Eingesandt und bearbeitet von Rechtsanwalt Tobias van der Ploeg, Schönerlinder Straße 6a, 16321 Bernau bei Berlin, Deutschland 